\title{
Experimental study of yarn friction slip and fabric shear deformation in yarn pull-out test
}

DOI:

10.1016/j.compositesa.2018.02.001

\section{Document Version}

Accepted author manuscript

Link to publication record in Manchester Research Explorer

\section{Citation for published version (APA):}

Bai, R., Li, W., Lei, Z., Ma, Y., Qin, F., Fang, Q., Chen, X., \& Chen, Y. (2018). Experimental study of yarn friction slip and fabric shear deformation in yarn pull-out test. Composites Part A: Applied Science and Manufacturing, 107, 529-535. https://doi.org/10.1016/j.compositesa.2018.02.001

\section{Published in:}

Composites Part A: Applied Science and Manufacturing

\section{Citing this paper}

Please note that where the full-text provided on Manchester Research Explorer is the Author Accepted Manuscript or Proof version this may differ from the final Published version. If citing, it is advised that you check and use the publisher's definitive version.

\section{General rights}

Copyright and moral rights for the publications made accessible in the Research Explorer are retained by the authors and/or other copyright owners and it is a condition of accessing publications that users recognise and abide by the legal requirements associated with these rights.

\section{Takedown policy}

If you believe that this document breaches copyright please refer to the University of Manchester's Takedown Procedures [http://man.ac.uk/04Y6Bo] or contact uml.scholarlycommunications@manchester.ac.uk providing relevant details, so we can investigate your claim.

\section{OPEN ACCESS}


Part A

Manuscript Number: JCOMA-17-2327R1

Title: Experimental study of yarn friction slip and fabric shear deformation in yarn pull-out test

Article Type: Research Paper

Keywords: A. Fabrics/textiles; B. Stress transfer; D. Non-destructive testing.

Corresponding Author: Professor Zhenkun Lei, Dr

Corresponding Author's Institution: Dalian Univ. of Technology

First Author: Ruixiang Bai

Order of Authors: Ruixiang Bai; Weikang Li; Zhenkun Lei, Dr; Yu Ma; Fuyong Qin; Qingchao Fang; X Chen; Yang Chen

Abstract: Single yarn pull-out test is a model experiment method to research the mechanical properties of fabric under impact. This study aims to understand the yarn pull-out mechanism and fabric shear deformation behavior of Kevlar 49 plain fabric by using the single yarn pull-out test combined with the digital image correlation method. The load-displacement curve contains typical physical phenomena such as crimp extension, crimp swap, frictional slip of yarn, and fabric deformation behavior. In the static friction stage, the pull-out load increased nonlinearly with the displacement, and the crimp extension of the pulled warp yarn occurred gradually. In the kinetic friction stage, the load decreased undulately until the warp was pulled out. Moreover, the fabric shear deformation sharply increased in the static friction stage, then decreased slowly during the kinetic friction stage. It was found that fabric shear deformation was still apparent after the yarn was completely pulled out.

Response to Reviewers: Please see the attached file "Response to Reviewer comment.doc" 


\section{Response to the Reviewer's Comments}

The authors appreciate the comments and the suggestions from the reviewers. The manuscript has been revised throughout. The responses are as follows:

\section{A list of change}

- Fig. 1 and 4 have been revised for better clarity and clearness.

- The spelling mistake of "wrap" has been revised to "wrap" throughout the manuscript.

- The revisions are highlighted in blue color.

\section{To Reviewer 1}

Q1. Figure 1 is worthy to exhibit since it is possible to identify dimensions and interweaving in the experimented textile. It seems to be a woven type textile. Referring to impact energy absorbing capacity and efficient energy transfer capability in ballistics area, it has been widely studied that interweaving (textile architecture or geometry) is a key factor. It has been also demonstrated that knitted type textiles are among those with better properties in this matter. Why did you use only woven type? Any chance to predict or evaluate any other geometry? Drapeabilty is also very important when energy dissipation comes; Woven fabrics are relatively stiff when compared with knitted fabrics which show the most interesting drape behavior. Therefore describe why woven is significant in ballistic studies.

Response: For a knitted fabric structure, as shown in Fig. R1(a), the impact energy dissipates in all directions, as shown in Fig. R1(b). Different to the knitted fabric, the energy transmission mainly spreads on the primary cross yarns of the plain woven fabric, as shown in Fig. R2. Due to the special structure of knitted fabric, the phenomenon of yarn pull-out does not exist in the ballistic impact process, and the energy dissipation caused by the relative sliding between the yarn compared with the strain energy and kinetic energy of the yarn can be neglected [R1].

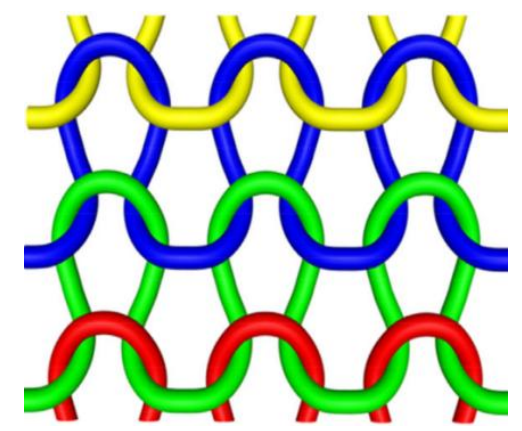

(a)

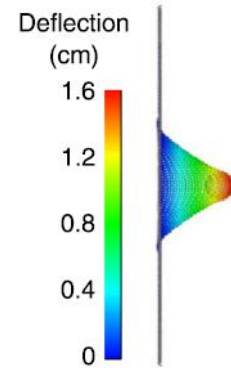

(b)

Fig. R1 (a) Knitted fabric structure and (b) simulated deflection under impact [R1]

Therefore, the interfacial modification to increase the friction between the yarns does not significantly improve the energy absorption capacity of the knitted fabric. Moreover, due to the special structure of the knitwear itself, a single yarn can not be completely extracted from the fabric, so such knitted fabric does not have the potential to perform the relevant type of experiment. 


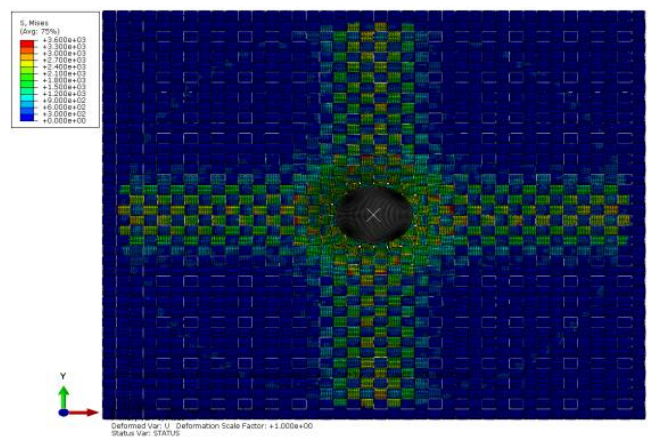

Fig. R2 Simulated Mises stress map for the plain woven fabric

The plain fabric is one of the simplest cross-weaved structure, which is basic for many woven type structures, such as 3D orthogonal woven structure, 3D interlocking weave structure, twill weave structure and satin weave structure, as shown in Fig. R3.

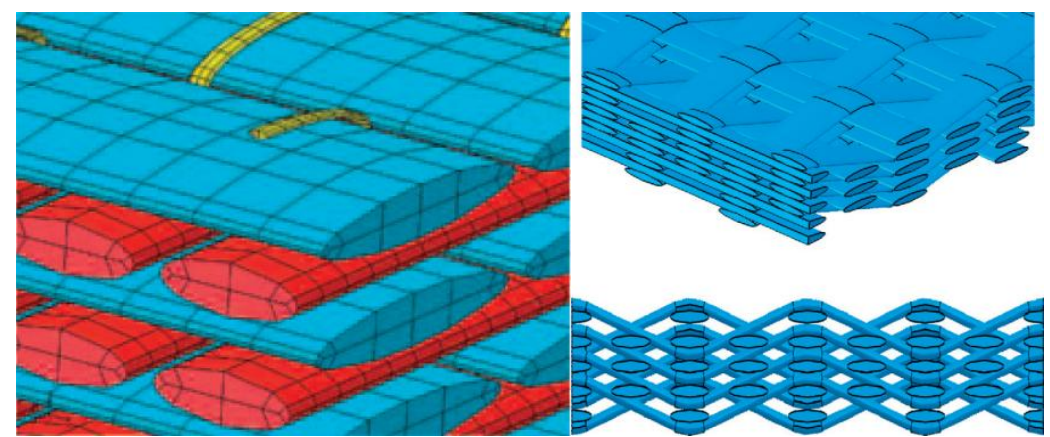

3-D orthogonal weave

3-D interlocking weave

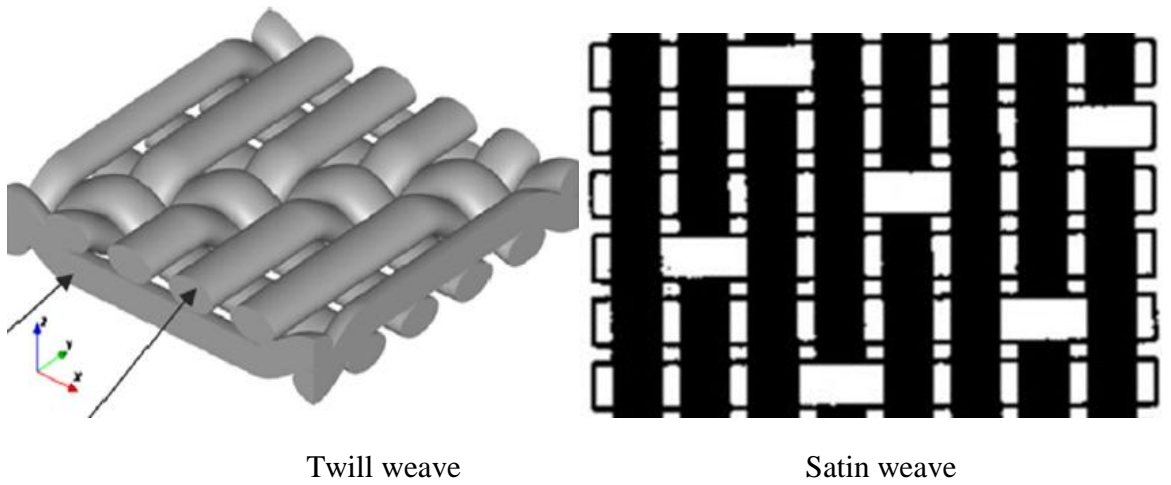

Fig. R3 Different woven structures

The evaluation of the impact resistance of bullet-proof fabrics not only depends on the energy absorption capacity but also on the degree of deformation of the fabrics after impact. In terms of ballistic protective materials, the conventional warp and weft knitted fabric structures are not used at all due to large transverse deformation the produce when receiving impacting load. According to the United States NIJ standard [R2], it allows maximum 44 mm backface signature (BFS), but the BSF of knitted fabrics will exceed that easily. One exception is the use of non-crimp multidirectional structures, in which the straight yarns in various directions are stitched together using a warp knitting structure. In this case, the warp knitting structure is not load-bearing. 
The plain woven structure not only has sufficient flexibility to meet the requirements of soft bullet-proof equipment, but also has certain resistance to deformation relative to the knitted fabric. A suitable level of flexibility is necessary for the ballistic panels, but the so-called drapeability is not needed for soft body armour. Therefore, the plain weave structure is more suitable for bullet-proof equipment and have great significance for bulletproof research.

[R1] Dani Liu, Daniel Christe, Bahareh Shakibajahromi, Chelsea Knittel, Nestor Casaneda, David Breen, Genevieve Dion, Antonios Kontsos. On the role of material architecture in the mechanical behavior of knitted textiles. International Journal of Solids and Structures. 109 (2017) 101-111.

[R2] Sarah V. Hart, Wendy Howe. Selection and application guide to personal body armor. NIJ Guide 100-01, 2001.

Q2. How the yarn pull out test is correlated to ballistic experimentation?

Response: The deformation and failure mechanisms during the ballistic impact of a flexible woven textile fabric are complicated involving multiple length and time scales affected by material behavior, projectile geometry-fabric interaction, impact velocity and boundary conditions. Some of the complex mechanisms observed in a fabric length scale impact experiments are: transverse shear and compression failure of the initial layers, pyramidal wave propagation, yarn pull out, inter-yarn friction, progressive fiber failure, slipping through of the sharp nosed projectiles, spreading and flattening of fibers and fibrillation. Lower length scale experiments are necessary to better understand these mechanisms and the associated energy absorption [R3].

The yarn pull-out test is used to model the fiber pull-out process during the ballistic penetration of the fabric, and to analyze the load transfer mechanism in the yarns and the shear deformation of the fabric. The yarn pull-out test is a quasi-static test whereas the ballistic impact is a dynamic process. However, it is found by many that the yarn pull-out test results correlates reasonably well with the dynamic behavior of the materials. Therefore, it is a foundation for the further interface modified ballistic fabric yarn drawing and ballistic penetration work, which is of great significance to improve the bullet-proof fabric performance and the optimal design of the soft bullet-proof equipment.

Micro Raman spectroscopy is a useful tool to investigate the yarn stress transfer in a fabric by yarn drawing test or out-of-plane push-out test. In our recent work [R4], the full-field stress distribution of a two-dimensional plain fabric was mapped using micro Raman spectroscopy through yarn push-out test, simulating a quasi-static projectile impact on the fabric. From the full-field fabric stress distribution, as shown in Fig. R4, it can be observed that there is a cross-shaped distribution of high yarn stress; this result would be helpful in further studies on load transfer on a fabric during a projectile impact.

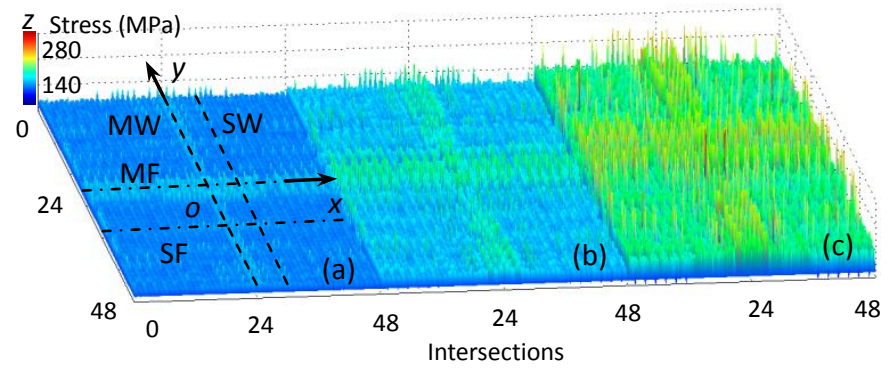


Fig. R4 Full-field stress distribution of circularly fixed fabric under different out-of-plane loads with displacements of (a) $6 \mathrm{~mm}$, (b) $12 \mathrm{~mm}$, and (c) $18 \mathrm{~mm}$.

[R3] S Sockalingam, SC Chowdhury, JW Gillespie, M Keefe. Recent advances in modeling and experiments of Kevlar ballistic fibrils, fibers, yarns and flexible woven textile fabrics-a review. Textile Research Journal. 2017, 87(8): 984-1010.

[R4] ZK Lei, FY Qin, QC Fang, RX Bai, W Qiu, X Chen. Full-field fabric stress measurement using micro Raman spectroscopy in yarn push-out test. Applied Optics. 2018, Accepted.

Q3. What about high speed impact measurement for energy dissipation?

Response: Energy absorption by a ballistic fabric can be measured via a perforation test, where the energy loss of the projectile is regarded as the same as the energy absorption of the fabric. During the high-speed impact of the ballistic fabric, the impact energy $\left(E_{p}\right)$ propagates predominantly in the direction of the primary yarn in contact with the projectile. Neglecting the secondary energy dissipation modes such as acoustic energy and thermal energy, the main modes of energy dissipation propagation are the strain energy $\left(E_{s}\right)$, the frictional energy loss $\left(E_{f}\right)$ between the yarns and the yarn break energy $\left(E_{d}\right)$ and kinetic energy $\left(E_{\mathrm{k}}\right)$ obtained after the impact of the yarn. It is expressed as follows:

$$
E_{p}=E_{s}+E_{f}+E_{d}+E_{k} .
$$

The impact energy of the projectile mainly propagates along the primary yarn, propagating to the secondary yarn through the intersection with the secondary yarn, and thus the energy is diffused throughout the ballistic fabric. Among them, the primary yarn strain energy, fracture energy, the friction energy between the primary yarn and the secondary yarn, the kinetic energy of the primary yarn, accounting for the vast majority of energy-dissipated during the bullet-proof fabric ballistic penetration. It can be seen that it is necessary to model the yarn pull-out process during ballistic penetration into a yarn pull-out test to analyze the friction energy dissipation mechanism. 


\title{
Experimental study of yarn friction slip and fabric shear deformation in yarn
}

\section{pull-out test}

\author{
Ruixiang Bai ${ }^{1}$, Weikang $\mathrm{Li}^{1}$, Zhenkun $\mathrm{Lei}^{{ }^{1}}{ }^{*}$, Yu Ma ${ }^{1}$, Fuyong Qin ${ }^{1}$, Qingchao Fang ${ }^{1}$, Xiaogang Chen $^{2}$, Yang Chen ${ }^{1}$ \\ 1 State Key Laboratory of Structural Analysis for Industrial Equipment, Dalian University of Technology, Dalian 116024, China \\ 2 School of Materials, The University of Manchester, Manchester M13 9PL, UK \\ *Correspondent author: leizk@163.com
}

\begin{abstract}
Single yarn pull-out test is a model experiment method to research the mechanical properties of fabric under impact. This study aims to understand the yarn pull-out mechanism and fabric shear deformation behavior of Kevlar 49 plain fabric by using the single yarn pull-out test combined with the digital image correlation method. The load-displacement curve contains typical physical phenomena such as crimp extension, crimp swap, frictional slip of yarn, and fabric deformation behavior. In the static friction stage, the pull-out load increased nonlinearly with the displacement, and the crimp extension of the pulled warp yarn occurred gradually. In the kinetic friction stage, the load decreased undulately until the warp was pulled out. Moreover, the fabric shear deformation sharply increased in the static friction stage, then decreased slowly during the kinetic friction stage. It was found that fabric shear deformation was still apparent after the yarn was completely pulled out.
\end{abstract}

Key words: A. Fabrics/textiles; B. Stress transfer; D. Non-destructive testing.

\section{Introduction}

Currently, there are three types of body armor: hard body, soft body, and hard-soft composite. Composite body armor is the focus of research, because it can prevent the secondary damage of shrapnel, and it is soft and lightweight [1]. High-quality body armor material must have good energy absorbing capacity, and fast and efficient energy transfer capability [2]. Kevlar fiber is a type of aramid composite developed by DuPont in the 1960s; it has high specific strength and modulus, good toughness, high temperature resistance, and high energy storage capacity and energy transfer efficiency. Kevlar fabric is woven with Kevlar yarn, and each yarn consists of hundreds or thousands of fibers. Thus, it is an ideal composite fiber for manufacturing bullet-proof clothing [3]. The evaluation of the impact resistance of bullet-proof fabrics not only depends on the energy absorption capacity but also on the degree of deformation of the fabrics after impact. In terms of ballistic protective materials, the conventional warp and weft knitted fabric structures are not used at all due to large transverse deformation the produce when receiving impacting load. Due to the special structure of knitted fabric, the phenomenon of yarn pull-out does not exist in the ballistic impact process, and the energy dissipation caused by the relative sliding between the yarn compared with the strain energy and kinetic energy of the yarn can be neglected [4]. According to the United States NIJ standard [5], it allows maximum $44 \mathrm{~mm}$ backface signature (BFS), but the BSF of knitted fabrics will exceed that easily. The plain woven structure not only has sufficient flexibility to meet the requirements of soft bullet-proof equipment, but also has certain resistance to deformation relative to the knitted fabric.

In the impact process, there are three types of transfers of the kinetic energy of a bullet: the kinetic energy of yarn, strain energy of yarn, and friction dissipation caused by frictional slip [6]. Of the three, friction force has an important role in the impact process. Some studies have shown that the fabric will absorb more energy with the increase of friction force; but when the friction force is too large, a premature rupture of the yarns may occur and reduce the energy absorbing ability of the fabric [6-9]. The main role of friction force during impact is to strengthen the interaction of yarns, and remote yarns can contribute to energy absorption. The moderate friction can 
improve energy absorption as well as delay fabric failure through increasing fabric deformation during impact [6]. In a low-speed impact, there are three main failure modes of fabric: yarn pull-out, local yarn fracture, and remote yarn failure [9]. Multi-ply fabric impact tests and numerical simulation showed that the front layers of fabric are more likely to be sheared out by a bullet, and the rear layers tend to be broken and have a larger deflection [10].

Owing to the high speed of a bullet at impact, fabric properties were studied by comparing the change in fabric state and bullet speed before and after impact, as it is difficult to monitor the deformation process of fabric during impact, and it is impossible to fully understand the friction mechanism. Lower length scale experiments are necessary to better understand these mechanisms and the associated energy absorption [11]. Some researchers developed the yarn pull-out test to simulate the process of yarn pulled out from fabric during impact [12]. The yarn pull-out test is a quasi-static test whereas the ballistic impact is a dynamic process. However, it is found by many that the yarn pull-out test results correlates reasonably well with the dynamic behavior of the materials. This method provides good insight into the fabric deformation process under impact, and is helpful in understanding friction and energy absorbing mechanisms.

Some research showed that yarn pull-out load is positively related to impact resistance, and that fabric with a high yarn pull-out load behaves better in an impact test [9, 13]. Zhu et al. [14] regarded the yarn pull-out process as the process of fiber pulled out from the matrix, and an analysis solution of load-displacement was established by considering the variations of yarn crimp extension and contact area. Bilisik et al. [15] studied the influence of fabric size and pull-out yarn amount as follows: the maximum yarn pull-out load is proportional to the longitudinal dimension of the fabric, while the transverse size has little effect on the maximum pull-out load. Moreover, when multiple yarns were pulled out at one time, the maximum load was larger than in the case of single yarn pull-out. Nilakantan et al. [16] found that the transverse preload is proportional to the maximum pull-out load. Dolatabadi et al. [17] studied fabric deformation under bias extension with the two-dimensional fast Fourier transform method, and the results showed that there is a critical shear angle during bias extension, which may be related to the geometric parameters of the fabric. Zhu et al. [18] discussed the deformation behavior of Kevlar 49 under bias extension; the results showed that fabric nonlinear properties and orthogonal behavior occurred under bias extension, and deformation reached $20 \%$ before fabric failure. Moreover, shear response has four distinct regions: linear elastic rotation, dissipative rotation, yarn compression, and shear locking. Bilisik et al. [19] studied the effects of yarn amount and width/length ratio on shear strength using the yarn pull-out test. However, the above works are statistical research on the relationships between load and displacement, and load and fabric geometric size. There is little research focusing on the distribution of friction force and the energy absorption mechanism at the mesoscopic level.

In this study, the yarn pull-out process was measured by the digital image correlation (DIC) method, and the load-displacement curve and fabric shear deformation were obtained. Combined with the mesoscopic geometry of fabric structure, the pull-out load, frictional force distribution, and energy absorption were investigated, and yarn crimp extension, crimp swap, frictional slip, and shear deformation were discussed.

\section{Geometric properties of fabric}

Kevlar 49 plain fabric (DuPont) was used in this study, and the basic properties are listed in Table 1.

Table 1 Material properties of fabric

\begin{tabular}{|l|c|c|c|c|}
\hline Type & Dtex & Density $\left(\mathrm{g} / \mathrm{cm}^{3}\right)$ & Number of fibers & Fiber diameter $(\mu \mathrm{m})$ \\
\hline Kevlar 49 & 1420 & 1.44 & 1000 & 12 \\
\hline
\end{tabular}

The micrograph of Kevlar 49 plain fabric is shown in Fig. 1(a); the fabric is woven alternately by warp yarn in the vertical direction and fill (weft) yarn in the horizontal direction. The figure shows a period cell structure consisting of $2 \times 2$ intersections of warp and fill yarns. Owing to the interaction of warp and fill yarns formed during 
the process of weaving, wavy crimp exists in the fabric under a relaxation condition. As shown in Figs. 1(b) and 1 (c), fill and warp yarns have different weave lengths and widths of $f_{a}, w_{a}$ and $f_{b}, w_{b}$, respectively. A period size of a cell is $\left(f_{a}+w_{b}\right) \times\left(f_{b}+w_{a}\right)$ in the vertical and horizontal directions, respectively.
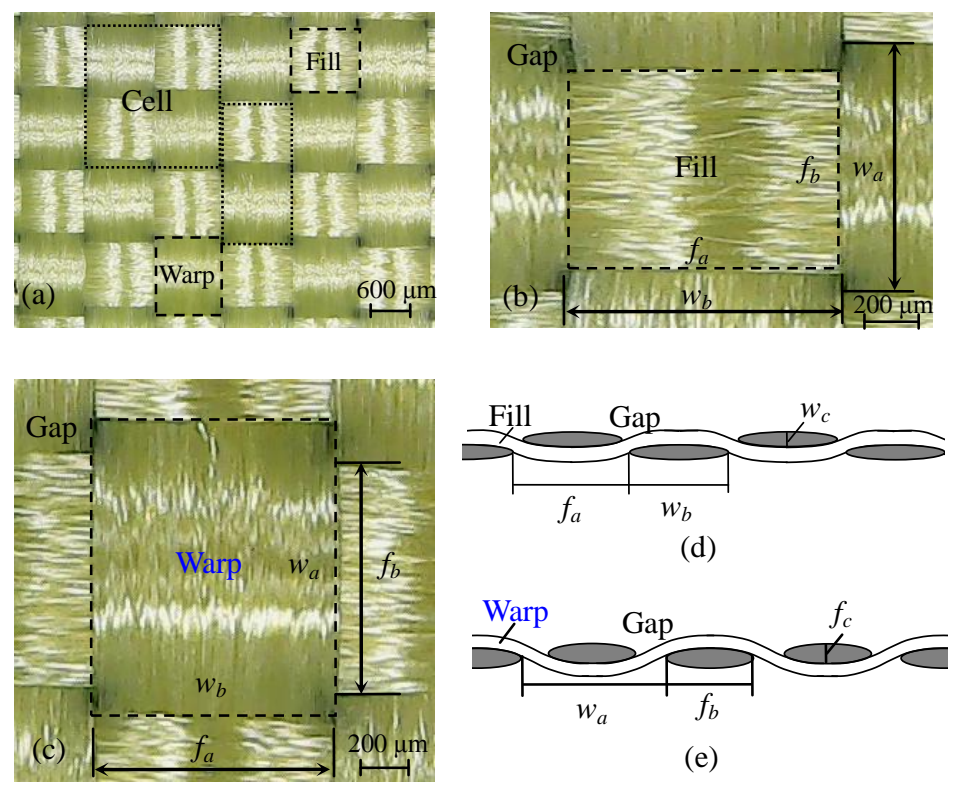

(d)

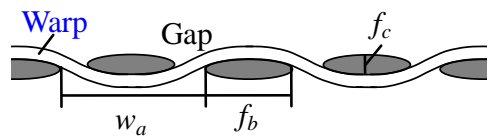

(e)

Fig. 1 (a)-(c) Geometric dimensions of Kevlar 49 plain woven fabric, (d) schematic diagram of warp trajectory with cross fill yarns, and (e) schematic diagram of fill trajectory with cross warp yarns

The abridged view of warp yarn trajectory with cross fill yarns and the fill yarn trajectory with cross warp yarns are shown in Figs. 1(d) and 1(e), respectively. The cross-sectional height of warp and fill yarns is $f_{c}$ and $w_{c}$, respectively. There is an overlapping region of warp and fill yarns, which is called the intersections of warp and fill yarns, and the intersection area is $S=f_{b} \times w_{b}$. In addition, there is a gap between the intersections, and the area of the gap is $s=\left(w_{b}-f_{a}\right) \times\left(w_{a}-f_{b}\right) / 2$. The geometric dimensions of warp and fill yarns are listed in Table 2 .

Table 2 Geometric dimensions of warp and fill yarns (unit: $\mathrm{mm}$ )

\begin{tabular}{|l|l|l|l|}
\hline Warp & $w_{a}=1.2$ & $w_{b}=1.1$ & $w_{c}=0.24$ \\
\hline Fill & $f_{a}=1.0$ & $f_{b}=1.0$ & $f_{c}=0.23$ \\
\hline
\end{tabular}

\section{Single yarn pull-out test}

\subsection{Sample and experiment process}

The test equipment used to conduct the experiment is shown in Fig. 2(a); two clamps are placed on a sliding rail to clip the two sides of fabric. Each clamp comprises three parts: front $\mathrm{u}$-shaped groove, back u-shaped groove, and the board between the two. Before the test, the fabric was placed between the board and the back groove, and secured by the bolts installed in the front groove. Hence, the fabric was clamped tightly between the board and the back groove. This design reduces boundary stress concentration and improves the accuracy of the experiment.

Before the experiment, the direction of the warp yarn is along the tension direction and the upper end of the warp yarn is loaded. The width and length of the sample are both $15 \mathrm{~cm}$. There are a total of 65 cells (130 intersections) along the warp direction and the same number of cells along the fill direction. There is no preload in the sample before the experiment. The middle warp yarn is pulled out using a universal testing machine (Instron 3345) and the pull-out speed is $100 \mathrm{~mm} / \mathrm{min}$.

Before the experiment, black markers were placed on the intersections of the pulled warp yarn and the 14 fill 
yarns in order to measure fabric deformation by DIC, as shown in Fig. 2(b). There were a total of 112 markers on the sample. During the experiment, a camera (AVT, F080b) was used to take photos with a frame rate of $5 \mathrm{fps}$, and the image size was $1024 \times 768$ pixels.
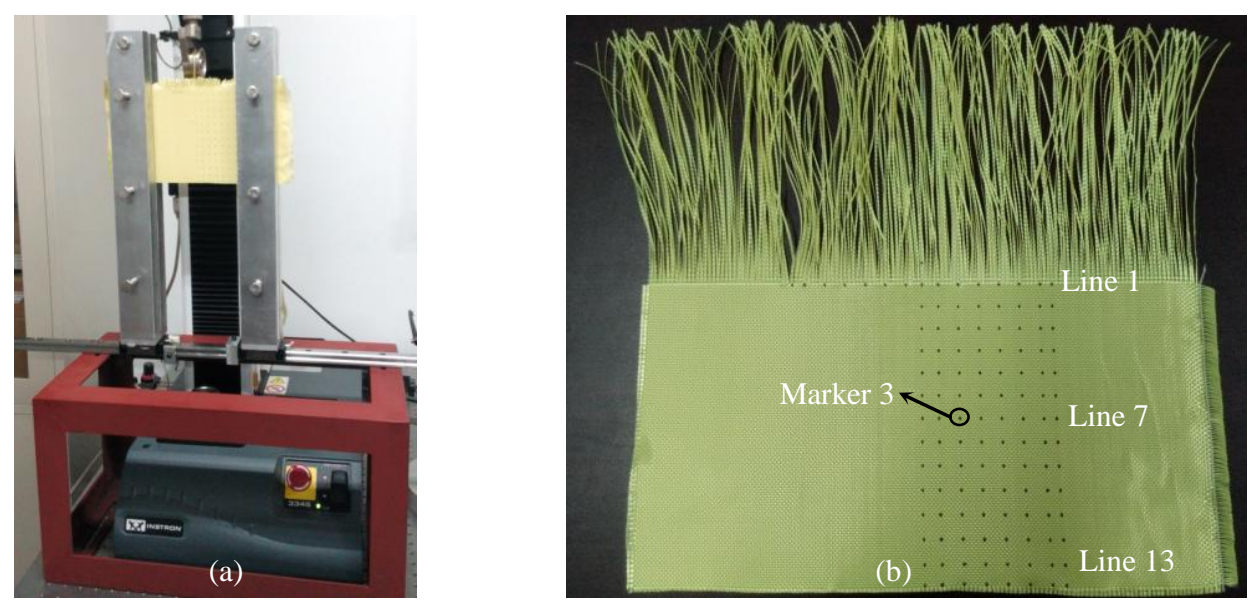

Fig. 2 (a) Experimental setup and (b) markers on fabric

\subsection{Pull-out load and displacement curve}

The load-displacement curve of the yarn pull-out test is shown in Fig. 3(a). There are two distinct regions: static friction and kinetic friction. In the static friction region, the pulled yarn had crimp extension and produced the static friction force among the intersections, which led to the fabric deformation. When the pull-out load reached the maximum, the pulled yarn began to slip in the kinetic friction region in Fig. 3(b).

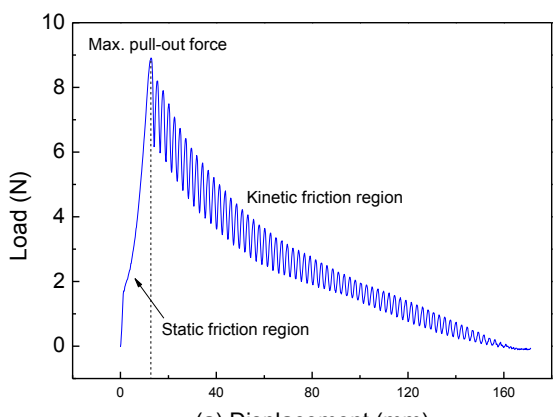

(a) Displacement ( $\mathrm{mm})$

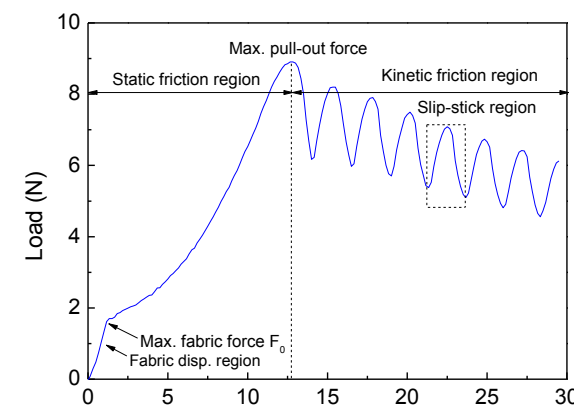

(b) Displacement $(\mathrm{mm})$

Fig. 3 (a) Typical curve of load and displacement during single yarn pull-out test and (b) slip-stick region

In the kinetic friction region, the load-displacement curve shows a classic sine-wave feature corresponding to a yarn crimp swap, where the pull-out load first increased and then decreased. The increase of pull-out load indicates that the bottom end of the pulled warp yarn is slipping into the adjacent intersection, while the decrease of pull-out load indicates the bottom end is slipping out of the current intersection. A sine wave corresponds to two intersections. When the pulled warp yarn slips through two intersections, it is a "slip in-out" process corresponding to the production of a sine wave in the load-displacement curve, shown as a dashed rectangle in Fig. 3(b). Therefore, the load-displacement curve comprises fabric shear deformation at a macroscopic scale and yarn deformation at a mesoscopic scale, which reflects a coupled physical phenomenon of yarn crimp extension, crimp swap, and friction slip.

At the beginning, the fabric sample produced overall deformation and the pull-out load varied linearly with displacement, owing to the structural adjustment of warp and fill yarns, as shown in Fig. 4(a). Next, the pulled warp yarn produced crimp extension, as shown in Fig. 4(b); the fabric sample produced shear deformation, and the 
pull-out load increased exponentially with displacement in the static friction stage.

After the crimp extension of the pulled warp yarn had finished, the kinetic friction began immediately, and the load-displacement curve decreased undulately until the pulled warp yarn completely slipped out of the fabric sample. During the kinetic friction stage, when the pulled warp yarn and cross fill yarns are in convex to convex contact, the pull-out load increases (Fig. 4(c)). When the pulled warp yarn and cross fill yarns are in convex to concave contact, the pull-out load decreases (Fig. 4(d)).

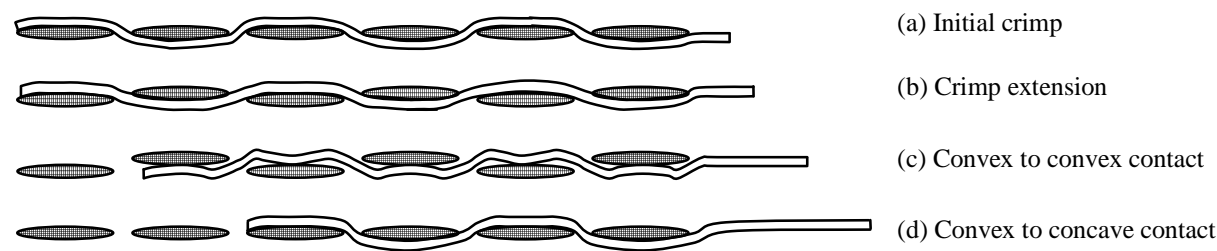

Fig. 4 Schematic diagram of single yarn pull-out process

\section{Results and discussion}

\subsection{Friction distribution during kinetic friction stage}

For simplicity, the first wave valley after the maximum static friction force is taken as the starting point; the first wave number is 1, and so on; and the last wave number is 65 . Extracting the load data of wave peak and wave valley, the distribution of pull-out load in the kinetic stage is shown in Fig. 5(a).
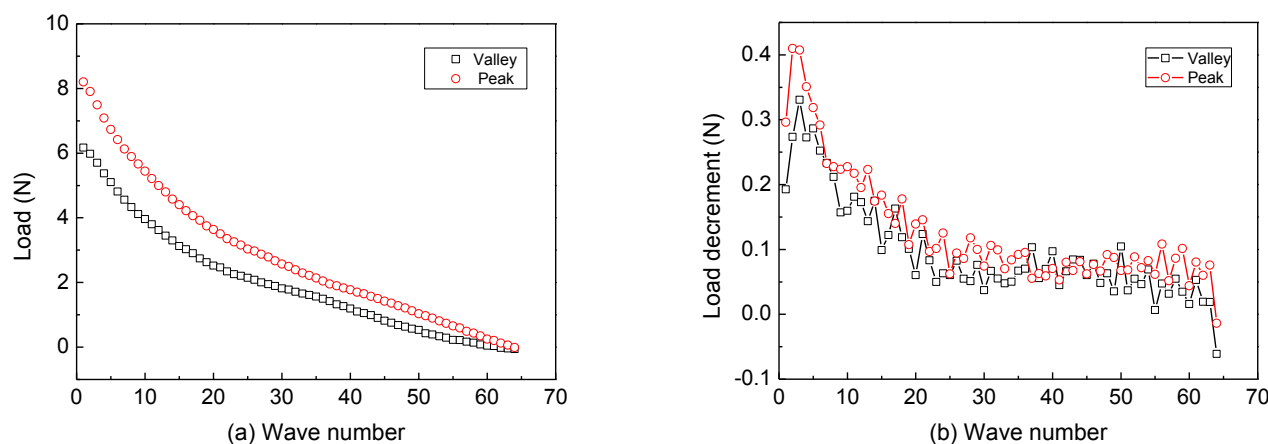

Fig. 5 (a) Pull-out load curves and (b) load decrement curves with wave number of peak and valley

In Fig. 5, the horizontal axis represents the wave number, while the vertical axis represents the pull-out load. The pull-out load curves of wave peak and wave valley have the same trend and are exponentially decreased. The pull-out loads decreased sharply at the beginning and then decreased slowly. Lastly, the curves converged.

The prior wave peak subtracts the posterior wave peak, and the prior wave valley subtracts the posterior wave valley; thus, the pull-out load decrement curves can be obtained, as shown in Fig. 5(b). The pull-out load decrement decreased sharply in the initial stage and decreased slowly. The wave peak and wave valley curves have the same trend.

The pull-out load is equal to the sum of the friction forces on all intersections, and the intersection number is reduced by two for a cell length or a wave period. Therefore, the pull-out load decrement in Fig. 5(b) is equal to the friction force on the pulled intersection. The load decrement decreased sharply in the initial stage, which means that the friction force is large at the initial intersections near the bottom of the fabric. The load decrement then decreased slowly, which indicates that the friction force has a nonuniform distribution and is related to the distance to the top of fabric.

\subsection{Energy absorbing mechanism}

The impact energy of the projectile mainly propagates along the main yarn, propagating to the secondary yarn 
through the intersection with the secondary yarn, and thus the energy is diffused throughout the ballistic fabric. Among them, the primary yarn strain energy, fracture energy, the friction energy between the primary yarn and the secondary yarn, the kinetic energy of the primary yarn, accounting for the vast majority of energy-dissipated during the bullet-proof fabric ballistic penetration. It can be seen that it is necessary to model the yarn pull-out process during ballistic penetration into a yarn pull-out test to analyze the friction energy dissipation mechanism.

In Fig. 3, the area under the pull-out load and displacement curve is the total energy of the test machine. Each wave area of the curve in the kinetic friction stage can be calculated by the sum of several sections; as shown in Fig. 6(a), there is a relationship between the absorbed energy and the wave number.
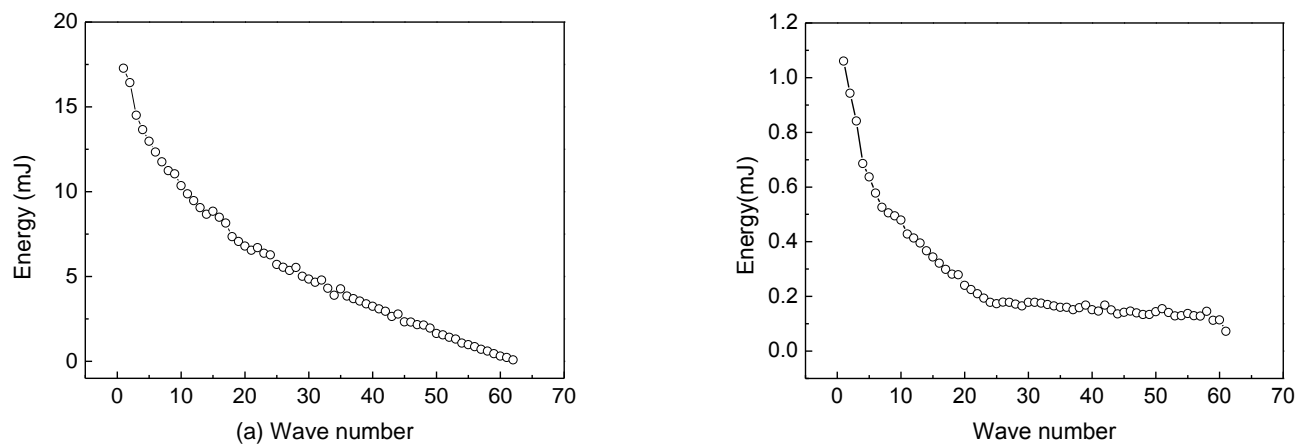

Fig. 6 (a) Absorbed energy curve and (b) energy decrement curve with wave number

As shown in Fig. 6(a), every point on the curve represents the sum of energy absorption for all intersections. The absorbed energy decreases exponentially with the intersection number as the yarn is pulling out. Similarly, the prior absorbed energy subtracts the posterior absorbed energy; thereafter, the energy absorption for each intersection can be obtained, as shown in Fig. 6(b). The absorbed energy decrement is equal to the friction energy for each intersection, which also has a nonuniform distribution. The absorbed energy decrement decreased sharply at the beginning intersections near the bottom of the fabric, and then became linear.

\subsection{Shear deformation behavior}

As the yarn pulled out, the fabric produced shear deformation. The shear angle $\theta$ is defined in this study as the angle between the fill yarn and the horizontal direction. A positive angle rotates in a counterclockwise direction; as shown in Fig. 2(a), the initial angle is $0^{\circ}$ before loading. As the symmetrical boundary and geometry conditions, only the shear deformation on the right side of the fabric was studied.

As shown in Fig. 2(b), there are 14 lines of markers along the fill yarns from top to bottom in order. Similarly, there are 7 markers on each line from left to right in order. For example, the marker " $7-3$ " is located at the 3 th point on the $7^{\text {th }}$ line. The displacement for each marker was measured by the DIC method; as shown in Fig. 7, there are displacement-time curves for the markers on the $1^{\text {st }}, 5^{\text {th }}, 10^{\text {th }}$, and $14^{\text {th }}$ lines.

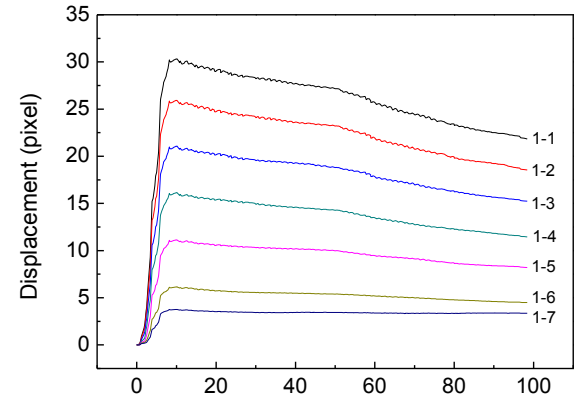

(a) Time (s)

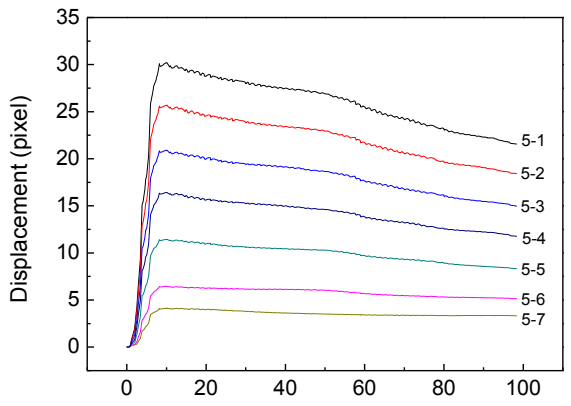

(b) Time(s) 

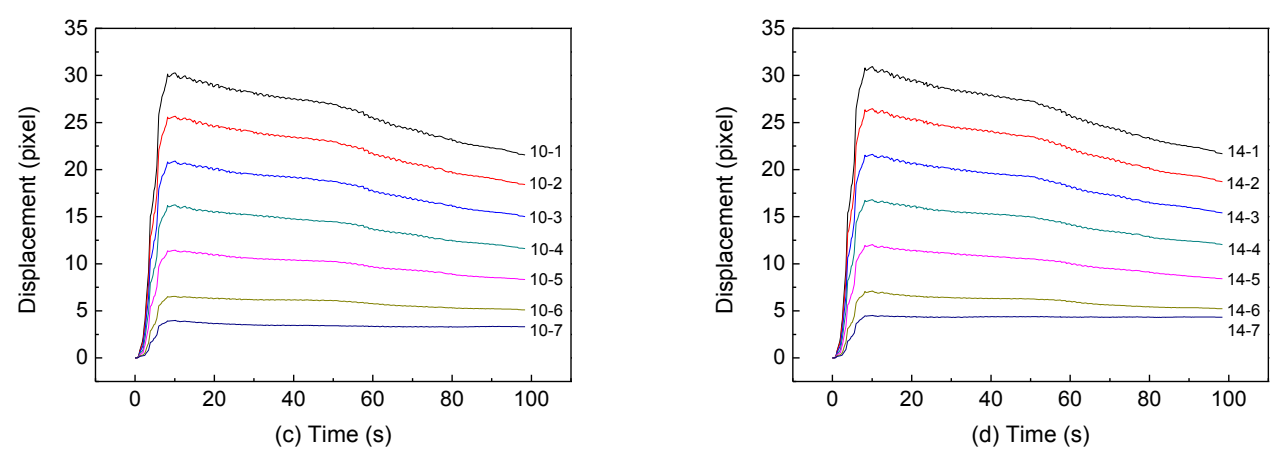

Fig. 7 Displacement with time curves of 7 markers on (a) line 1, (b) line 5, (c) line 10, and (d) line 14

As shown in Fig. 7, the displacement-time curves of 7 markers on different lines have a similar tendency. The displacement first increases sharply and then decreases slowly after reaching the maximum value. The marker on each line is closer to the pulled warp yarn; the rising rate or decline rate is higher. Moreover, all the markers on each line have a maximum displacement at $10.5 \mathrm{~s}$, which corresponds to the maximum load on the pull-out load and time curves (Fig. 3). This result means that the fabric overall displacement increased with the pull-out load in the static stage and decreased with the pull-out load in the kinetic stage. Obviously, the fabric still maintained a larger displacement as the yarn was completely pulled out, because the yarn crimp extended in the intersections.

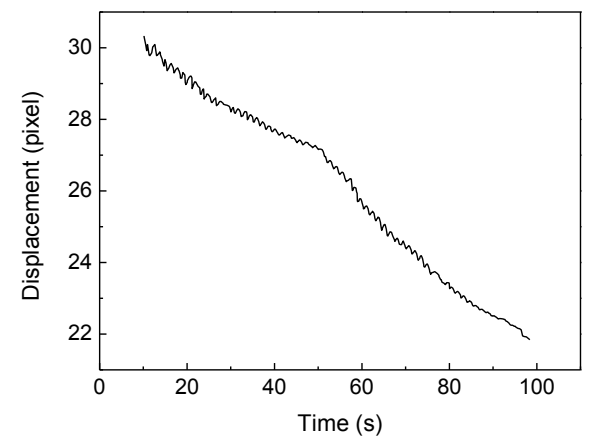

Fig. 8 Displacement with time curve of the marker 1-1 during kinetic friction

To further study fabric shear deformation, the displacement-time curve of the marker "1-1" in the kinetic stage is extracted, as shown in Fig. 8. It can be seen that marker displacement decreases undulately, which is coincident with the pull-out load and displacement curve (Fig. 3). Moreover, the coordinate positions of 7 markers on each line were fitted using a linear least square method; therefore, the slope (shear angle) for each line can be obtained, as shown in Fig. 9(a).
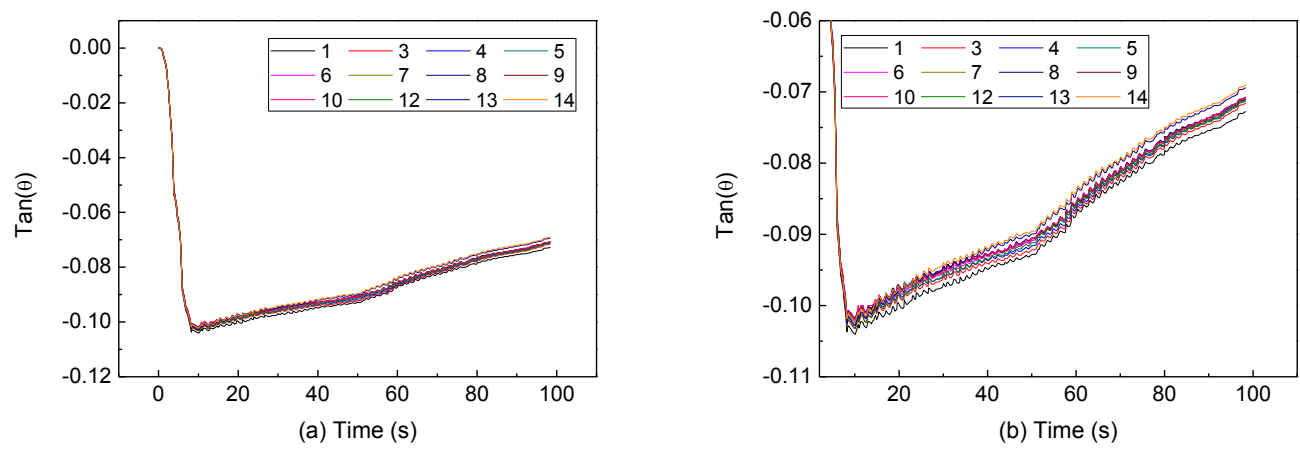

Fig. 9 (a) $\operatorname{Tan}(\theta)$ with time curve for different lines and (b) partial magnification 
It can be seen from Fig. 9(a) that the shear angles for different lines are very similar. In the initial stage, the absolute value of the shear angle increased rapidly and reached the maximum value at $10.5 \mathrm{~s}$. This result means that the fabric shear deformation reached the maximum value in the maximum static friction moment. Next, in the kinetic friction stage, the absolute value of the shear angle decreased gradually until the yarn was completely pulled out. This result means that the fabric remained at a larger deformation due to the yarn crimp extension. It is clear from Fig. 9(b) that the absolute value of the shear angle decreases in the dynamic friction phase, which corresponds to the oscillation of the pull-out force displacement curve.

\section{Conclusions}

The yarn pull-out test results show that the pull-out load and displacement curve comprises macroscopic fabric shear deformation and mesoscopic yarn deformation, and reflects yarn extension crimp, crimp swap, and friction slip. In the static friction stage, the pulled yarn produced the crimp extension; hence, the overall shear deformation of the fabric occurred under the pull-out load. In the kinetic friction stage, when the pulled warp yarn and the cross-fill yarns were in convex to convex contact, the pull-out load increased; when the pulled warp yarn and the cross-fill yarns were in convex to concave contact, the pull-out load decreased. Further study shows that the friction force on the pulled warp yarn was nonuniform, which decreased exponentially with the pull-out intersections of the fabric.

The fabric shear deformation was studied by the DIC method. The measurement results show that the maximum shear deformation of the fabric was achieved at the maximum friction force in the static friction stage. Moreover, in the kinetic friction stage, the shear angle decreased gradually. After the yarn was completely pulled out, the fabric maintained a relatively large deformation owing to yarn crimp extension.

\section{Acknowledgement}

This work was supported by National Natural Science Foundation of China (Nos. 11472070, 11772081, 11572070).

\section{References}

[1] Chen X. Modelling and predicting textile behaviour. Cambridge: Woodhead Publishing Ltd, 2010.

[2] Ajmer KD, Michael WD, Stephen AF, Kyle AS, Larry RL, Eric DW. Low velocity ballistic behavior of continuous filament knit aramid. Int J Impact Engin 2016; 96: 23-34.

[3] Carr DJ. Failure mechanisms of yarns subjected to ballistic impact. J Mater Sci Let 1999; 18(7): 585-588.

[4] Liu D, Christe D, Shakibajahromi B, Knittel C, Casaneda N, Breen D, Dion G, Kontsos A. On the role of material architecture in the mechanical behavior of knitted textiles. Int J Solids Struct 2017; 109: 101-111.

[5] Hart SV, Howe W. Selection and application guide to personal body armor. NIJ Guide 100-01, 2001.

[6] Duan Y, Keefe M, Bogettic TA, Cheeseman BA. Modeling friction effects on the ballistic impact behavior of a single-ply high-strength fabric. Int J Impact Engin 2005; 31(8): 996-1012.

[7] Zhou Y, Chen X, Wells G. Influence of yarn gripping on the ballistic performance of woven fabrics from ultra-high molecular weight polyethylene fibre. Compos Part B 2014; 62(62): 198-204.

[8] Bilisik K. Properties of yarn pull-out in para-aramid fabric structure and analysis by statistical model. Compos Part A 2011; 42(12): 1930-1942.

[9] Bilisik K, Korkmaz M. Multilayered and multidirectionally-stitched aramid woven fabric structures: experimental characterization of ballistic performance by considering the yarn pull-out test. Textile Res J 2010; 80(16): 1697-1720. 
[10] Chen X, Zhou Y, Wells G. Numerical and experimental investigations into ballistic performance of hybrid fabric panels. Compos Part B 2014; 58(3): 35-42.

[11] Sockalingam S, Chowdhury SC, Gillespie JW, Keefe M. Recent advances in modeling and experiments of Kevlar ballistic fibrils, fibers, yarns and flexible woven textile fabrics - a review. Textile Res J 2017; 87: 984-1010.

[12] Bazhenov S. Dissipation of energy by bulletproof aramid fabric. J Mater Sci 1997; 32(15): 4167-4173.

[13] Dong Z, Sun CT. Testing and modeling of yarn pull-out in plain woven Kevlar fabrics. Compos Part A 2009; 40(12): 1863-1869.

[14] Zhu D, Soranakom C, Mobasher B, Rajan SD. Experimental study and modeling of single yarn pull-out behavior of Kevlar 49 fabric. Compos Part A 2011; 42(7): 868-879.

[15] Bilisik K, Korkmaz M. Single and multiple yarn pull-outs on aramid woven fabric structures. Textile Res J 2010; 81(8): 847-864.

[16] Nilakantan G, Gillespie JW. Yarn pull-out behavior of plain woven Kevlar fabrics: Effect of yarn sizing, pullout rate, and fabric pre-tension. Compos Struct 2013; 101(15): 215-224.

[17] Dolatabadi MK, Kovař R, Linka A. Geometry of plain weave fabric under shear deformation. Part I: measurement of exterior positions of yarns. Textile Res J 2009; 100(4): 368-380.

[18] Zhu D, Mobasher B, Vaidya A, Rajan SD. Mechanical behaviors of Kevlar 49 fabric subjected to uniaxial, biaxial tension and in-plane large shear deformation. Compos Sci Technol 2013; 74(4): 121-130.

[19] Bilisik K. Experimental determination of fabric shear by yarn pull-out method. Textile Res J 2011; 82(10): 1050-1064. 


\section{List of Tables}

Table 1 Material properties of fabric

\begin{tabular}{|l|c|c|c|c|}
\hline Type & Dtex & Density $\left(\mathrm{g} / \mathrm{cm}^{3}\right)$ & Number of fibers & Fiber diameter $(\mu \mathrm{m})$ \\
\hline Kevlar 49 & 1420 & 1.44 & 1000 & 12 \\
\hline
\end{tabular}

Table 2 Geometric dimensions of warp and fill yarns (unit: $\mathrm{mm}$ )

\begin{tabular}{|l|l|l|l|}
\hline Warp & $w_{a}=1.2$ & $w_{b}=1.1$ & $w_{c}=0.24$ \\
\hline Fill & $f_{a}=1.0$ & $f_{b}=1.0$ & $f_{c}=0.23$ \\
\hline
\end{tabular}

\section{Captions of Figures}

Fig. 1 (a)-(c) Geometric dimensions of Kevlar 49 plain woven fabric, (d) schematic diagram of warp trajectory with cross fill yarns, and (e) schematic diagram of fill trajectory with cross warp yarns

Fig. 2 (a) Experimental setup and (b) markers on fabric

Fig. 3 (a) Typical curve of load and displacement during single yarn pull-out test and (b) slip-stick region

Fig. 4 Schematic diagram of single yarn pull-out process

Fig. 5 (a) Pull-out load curves and (b) load decrement curves with wave number of peak and valley

Fig. 6 (a) Absorbed energy curve and (b) energy decrement curve with wave number

Fig. 7 Displacement with time curves of 7 markers on (a) line 1, (b) line 5, (c) line 10, and (d) line 14

Fig. 8 Displacement with time curve of the marker 1-1 during kinetic friction

Fig. 9 (a) $\operatorname{Tan}(\theta)$ with time curve for different lines and (b) partial magnification 